\title{
Comparison between CNA and energetic electron precipitation: simultaneous observation by Poker Flat Imaging Riometer and NOAA satellite
}

\author{
Y.-M. Tanaka ${ }^{1}$, M. Ishii ${ }^{1}$, Y. Murayama ${ }^{1}$, M. Kubota ${ }^{1}$, H. Mori ${ }^{1}$, M.-Y. Yamamoto $^{2}$, A. Kadokura ${ }^{3}$, \\ D. Lummerzheim ${ }^{4}$, J. Desrochers ${ }^{4}$, and D. S. Evans ${ }^{5}$ \\ ${ }^{1}$ National Institute of Information and Communications Technology, Japan \\ ${ }^{2}$ Kochi University of Technology, Japan \\ ${ }^{3}$ National Institute of Polar Research, Japan \\ ${ }^{4}$ Geophysical Institute, University of Alaska Fairbanks, USA \\ ${ }^{5}$ NOAA Space Environment Center, Boulder, Colorado, USA
}

Received: 17 November 2004 - Revised: 14 March 2005 - Accepted: 16 March 2005 - Published: 27 July 2005

Part of Special Issue "Atmospheric studies by optical methods"

\begin{abstract}
The cosmic noise absorption (CNA) is compared with the precipitating electron flux for 19 events observed in the morning sector, using the high-resolution data obtained during the conjugate observations with the imaging riometer at Poker Flat Research Range (PFRR; $65.11^{\circ} \mathrm{N}, 147.42^{\circ} \mathrm{W}$ ), Alaska, and the low-altitude satellite, NOAA 12. We estimate the CNA, using the precipitating electron flux measured by NOAA 12, based on a theoretical model assuming an isotropic pitch angle distribution, and quantitatively compare them with the observed CNA. Focusing on the eight events with a range of variation larger than $0.4 \mathrm{~dB}$, three events show high correlation between the observed and estimated CNA (correlation coefficient $\left.\left(r_{0}\right)>0.7\right)$ and five events show low correlation $\left(r_{0}<0.5\right)$. The estimated CNA is often smaller than the observed CNA ( $72 \%$ of all data for 19 events), which appears to be the main reason for the low-correlation events. We examine the assumption of isotropic pitch angle distribution by using the trapped electron flux measured at $80^{\circ}$ zenith angle. It is shown that the CNA estimated from the trapped electron flux, assuming an isotropic pitch angle distribution, is highly correlated with the observed CNA and is often overestimated ( $87 \%$ of all data). The underestimate (overestimate) of CNA derived from the precipitating (trapped) electron flux can be interpreted in terms of the anisotropic pitch angle distribution similar to the loss cone distribution. These results indicate that the CNA observed with the riometer may be quantitatively explained with a model based on energetic electron precipitation, provided that the pitch angle distribution and the loss cone angle of the electrons are taken into account.
\end{abstract}

Correspondence to: Yoshimasa Tanaka

(ytanaka@nict.go.jp)
Keywords. Energetic particles, precipitating - Energetic particles, trapped - Ionosphere-magnetosphere interactions

\section{Introduction}

The auroral electron precipitation in the polar region plays an important role in the dynamics, energetics, and composition of the upper atmosphere. For several decades, the precipitating electrons with energies lower than $20 \mathrm{keV}$ have been extensively studied. Low-energy electron precipitation causes the auroral optical emission in the ionospheric $\mathrm{E}$ and $\mathrm{F}$ regions, and thus, optical measurements have been used to obtain the spatial structure, energy flux, and characteristic energy of the precipitating electrons (e.g. Semeter et al., 2001).

The precipitating electrons with medium energies between $20 \mathrm{keV}$ and a few $\mathrm{MeV}$ have been investigated from satellite measurements (Hartz and Brice, 1967; McDiarmid et al., 1975; Codrescu et al., 1997). Codrescu et al. (1997) presented the global patterns of precipitating electrons with energies between $30 \mathrm{keV}$ to a few $\mathrm{MeV}$, using flux data from the TIROS/NOAA satellites, and demonstrated that the mediumenergy electrons significantly affect the composition of the middle atmosphere. However, these global patterns are a statistical average of data collected during many satellite passes, and it is difficult for satellites to characterize individual events because individual satellite measurements are not able to separate the spatial and temporal variations in the precipitation.

The riometer has also been used to obtain information on medium-energy precipitating electrons. It measures the cosmic noise absorption (CNA), which is thought to be mainly caused by an increase in the electron density of the 
ionospheric $\mathrm{D}$ region, as produced by precipitating electrons with energies between $10 \mathrm{keV}$ and $100 \mathrm{keV}$. In particular, an imaging riometer (or a network of single-beam riometers) can reveal the two-dimensional structure of CNA to a relatively high time resolution and it can be used to separate the spatial and temporal variations in energetic electron precipitation for case studies. In addition, riometer observations cost much less than satellite measurements.

Although a riometer is a useful instrument for measuring medium-energy electrons, the relationship between CNA and precipitating electrons has yet to be clarified. So far, several authors have reported a good correlation between the CNA observed with broad-beam riometers and precipitating medium-energy electrons, by comparing with data from the low-altitude satellites (Maehlum and O'Brien, 1963; Hargreaves and Sharp, 1965; Parthasarathy et al., 1966; Jelly and Brice, 1967) and from a geostationary satellite (Collis et al., 1983, 1984; Collis and Korth, 1985). Some of them have also shown an empirical relationship between CNA and precipitating medium-energy electrons measured at low altitude (Hargreaves and Sharp, 1965; Parthasarathy et al., 1966) and at geosynchronous orbit (Collis et al., 1983). However, the broad-beam riometers used in these studies were incapable of measuring the fine structure of CNA due to their wide beam, and thus the relationship between CNA and the precipitating electron flux was spatially and temporally averaged. In addition, these previous studies include an ambiguity in the relationship between CNA and the electron flux, caused by the discrepancy in the locations of the absorption and the satellite footprint.

In this paper, we compare CNA with the precipitating electron flux at high spatial and temporal resolutions, by taking advantage of the imaging (narrow-beam) riometer at Poker Flat Research Range (PFRR; 65.11 ${ }^{\circ} \mathrm{N}, 147.42^{\circ} \mathrm{W}$ ), Alaska, and the low-altitude satellite, NOAA 12 . To reduce the above-mentioned ambiguities, we trace the magnetic field line from NOAA 12 to the footprint at $90 \mathrm{~km}$ and carefully pick up CNA data measured on the beam closest to the footprint. We estimate CNA from the precipitating electron flux as measured by NOAA 12 and quantitatively compare them with the CNA observed by the imaging riometer.

\section{Instrumentation}

The NOAA 12 satellite is in a sun-synchronous, nearcircular, polar orbit with an inclination of about $98.7^{\circ}$ (Raben et al., 1995). Its altitude and orbital period are approximately $815 \mathrm{~km}$ and $102 \mathrm{~min}$, respectively. It passes over PFRR around 06:30 MLT (16:30 MLT) during its southbound (northbound) orbit. We used the electron flux data from the Medium Energy Proton and Electron Detector (MEPED) and the Total Energy Detector (TED), which are a solid-state detector and an electrostatic analyzer, respectively. The MEPED has two detectors looking at about $0^{\circ}$ and $80^{\circ}$ zenith angles, corresponding to pitch angles $(\alpha)$ of about $0^{\circ}-25^{\circ}$ and $65^{\circ}-95^{\circ}$ at high latitudes, and measures integral counts of incident electrons with energies greater than $30 \mathrm{keV}, 100 \mathrm{keV}$, and $300 \mathrm{keV}$ once every $2 \mathrm{~s}$. For convenience, we will define the terms "MEPED-0" and "MEPED$90^{\circ} "$ to denote these two detectors looking at $0^{\circ}$ and $80^{\circ}$ zenith angles. The TED has two detectors that view at $0^{\circ}$ and $30^{\circ}$ zenith angles and each detector has 11 energy channels covering the energy range from $300 \mathrm{eV}$ to $20 \mathrm{keV}$. The TED identifies the energy channel that accumulates the greatest number of counts during the energy sweep, and the identified channel number and the accumulated counts are continuously telemetered every $2 \mathrm{~s}$. Assuming that the energy deposition to the atmosphere occurs at a $100-\mathrm{km}$ altitude, the loss cone angle is approximately $59^{\circ}$ at $815 \mathrm{~km}$ over PFRR. Therefore, the electrons measured by the MEPED $-0^{\circ}$ are completely within the loss cone and deposit the energy in the atmosphere, while the electrons measured by the MEPED$90^{\circ}$ are outside the loss cone and are trapped. Both TED sensors measure electrons within the loss cone.

The imaging riometer at PFRR features a $16 \times 16$-element square array that consists of 256 crossed-dipole antennas, aligned along the magnetic latitude and longitude. This imaging riometer provides a high-resolution, twodimensional image of CNA at the $38.2 \mathrm{MHz}$ frequency every second. Phasing synthesis by the Butler matrix yields 208 effective beams within the $70^{\circ}$ zenith angle, corresponding to $6^{\circ}$ in the two-way half-power width around the beam direction and $7^{\circ}$ in the beam interval. The viewing area is $400 \times 400 \mathrm{~km}^{2}$ at a $90-\mathrm{km}$ altitude and the horizontal spatial resolution is approximately $11 \mathrm{~km}$ around the local zenith. The observational ambiguity of CNA due to the system noise was examined and it did not have a great influence on the results in this paper. The technique of gathering data with this imaging riometer has been summarized by Murayama et al. (1997).

\section{Estimate of CNA using precipitating electron flux}

The events for the data analysis were selected as follows: we listed the time intervals in which the footprint of NOAA 12 at a $120-\mathrm{km}$ altitude passed across a box of $3^{\circ} \times 5^{\circ}$ geographic latitude and longitude centered at PFRR $\left(65.11^{\circ} \mathrm{N}\right.$, $147.42^{\circ} \mathrm{W}$ ) during the period from October 1995 through April 1998. For each interval, CNA data was sampled on the riometer beam closest to the satellite footprint at $90 \mathrm{~km}$ every $2 \mathrm{~s}$, resulting in a CNA event that consisted of about 30 data points $(60 \mathrm{~S})$. We selected the CNA events that had magnitudes of more than $0.4 \mathrm{~dB}$ at maximum. The CNA events coincident with the solar proton events were removed. Since the electron flux from the TED was very low in the afternoon sector over PFRR, the events observed in the afternoon sector were not used for data analysis. As a result, 19 events observed in the morning sector between 5.8 MLT and 7.1 MLT were selected.

The energy spectrum of the precipitating electrons is derived from the electron flux measured by the TED and MEPED pointed toward the zenith every $2 \mathrm{~s}$. The double 
Maxwellian distribution function is applied for the spectrum, which can be used to represent the electron flux in the magnetosphere (Garrett et al., 1981). We also examined a kappa distribution for the energy spectrum and confirmed that the results obtained with it were not so much different from the double Maxwellian distribution. The differential energy flux in the double Maxwellian distribution is given by the following equation.

$$
\begin{aligned}
& f(E)=f_{1}(E)+f_{2}(E)= \\
& A_{1} E^{2} \exp \left(-\frac{E}{E_{1}}\right)+A_{2} E^{2} \exp \left(-\frac{E}{E_{2}}\right),
\end{aligned}
$$

where $f_{1}(E)$ and $f_{2}(E)$ are the low-energy and high-energy components of the spectrum, respectively. $E_{1}$ and $E_{2}$ are the characteristic energies for the two components.

The low-energy component is derived from the two TED data, that is, the energy channel in which the maximum count was accumulated and the differential energy flux at that channel $\left(f_{\max }\right)$. The function $f_{1}(E)$ peaks at the energy $E=2 E_{1}\left(=E_{P}\right)$, where the peak energy $E_{P}$ is assumed to be the center energy of the maximum channel. Since the maximum peak in the $f(E)$ function agrees with $f_{\max }$ at the peak energy (i.e. $\left.f\left(E_{P}\right)=f_{1}\left(E_{P}\right)+f_{2}\left(E_{P}\right)=f_{\max }\right), A_{1}$ can be represented by a function of $A_{2}$ and $E_{2}$ from Eq. (1). It should be noted that the peak energy in $f(E)$ was assumed to correspond to that in $f_{1}(E)$.

$A_{2}$ and $E_{2}$ in the high-energy component are obtained from the MEPED data. First, the integral fluxes in the three energy bands, $>30 \mathrm{keV},>100 \mathrm{keV}$, and $>300 \mathrm{keV}$, are converted into differential fluxes in two energy bands, $30-100 \mathrm{keV}$ and $100-300 \mathrm{keV}$. Then, the differential energy fluxes are calculated by multiplying the differential flux by the mean energy of each energy band (i.e. $65 \mathrm{keV}$ for $30-100 \mathrm{keV}$ and $200 \mathrm{keV}$ for $100-300 \mathrm{keV}$ ). Finally, $A_{2}$ and $E_{2}$ are determined through an iterative procedure of the least-squares fitting so that the two differential energy fluxes derived from the MEPED are consistent with the energy fluxes integrated over energy from $30 \mathrm{keV}$ to $100 \mathrm{keV}\left(\int_{30 \mathrm{keV}}^{100 \mathrm{keV}} f(E) d E\right)$ and from $100 \mathrm{keV}$ to $300 \mathrm{keV}$ $\left(\int_{100 \mathrm{keV}}^{300 \mathrm{kV}} f(E) d E\right)$, respectively.

With the foregoing energy spectrum, the CNA generated by electron precipitation is estimated as follows: first, the energy deposition rate of precipitating electrons is calculated at each altitude according to the method given by Jackman et al. (1980). In the calculation, the MSIS-90 is used as the atmospheric model and an isotropic pitch angle distribution is assumed. The ion pair production rate $q(h)$ at an altitude of $h$ is obtained by dividing the energy deposition rate by the average ionization energy of $35 \mathrm{eV}$. Then, under the quasiequilibrium condition, the electron density $N_{e}(h)$ is given by

$N_{e}(h)=\sqrt{q(h) / \alpha_{e f f}(h)}$, where $\alpha_{e f f}(h)$ is the effective recombination coefficient as a function of $h$. Finally, CNA is calculated using the following equation;

$A=\int_{0}^{\infty} a(h) N_{e}(h) d h$,

where $A$ is CNA in $\mathrm{dB}$ and $a(h)$ is termed the specific absorption defined as the radio absorption $(\mathrm{dB})$ per kilometer due to a single electron per cubic centimeter. In this paper, we utilize the values in Fig. 15 by Hargreaves and Devlin (1990) for the effective recombination coefficient $\left(\alpha_{e f f}(h)\right)$ and the specific absorption $(a(h))$. The specific absorption $a(h)$ is multiplied by a factor of $(30.0 / 38.2)^{2}$ to adjust their calculation (frequency: $30 \mathrm{MHz}$ ) to ours (frequency: $38.2 \mathrm{MHz}$ ). The uncertainty of CNA due to the assumed $\alpha_{e f f}(h)$ is described in Sect. 5.

\section{Results}

Figure 1 shows a typical event observed from 17:20:06 to 17:21:08 UT on 28 February 1998. This CNA event was accompanied by a substorm, which started around 16:50 UT, with an $A E$ index of about $500 \mathrm{nT}$ at maximum, at 16:59 UT. The geomagnetic condition in this interval was moderately disturbed, with a $K_{P}$ index of $3+$. Each panel exhibits a CNA image sampled every $10 \mathrm{~s}$, in which the magnetic north is toward the top and east is toward the right, as one sees the ionosphere from above. The white cross on the images denotes the footprint of NOAA 12 at $90 \mathrm{~km}$ and the scintillations labeled by the letters, $R_{1}$ and $R_{2}$, are radio stars, Cassiopeia$\mathrm{A}$ and Cygnus-A, respectively. These images show the enhanced CNA region that stretches in the east-west direction. The enhanced CNA region has the relatively sharp boundary at higher latitude, while the boundary is more diffuse towards lower latitude. The fine structure of enhanced CNA is also seen around the zenith, except for the radio stars. This spatial structure is stable throughout the interval. The effect of the radio stars on the CNA at the satellite footprint was small during this interval. The CNA data collected when the satellite footprint was affected by the radio stars was not used for the analysis.

Figure 2a shows the precipitating electron flux measured by the MEPED- $0^{\circ}$ in this interval. The solid triangles in Fig. 2b show CNA observed simultaneously at the beam closest to the satellite footprint. It can be seen that the enhanced CNA observed during 17:20:20-17:20:44 UT is accompanied by an enhancement in electron flux in the 30 $100 \mathrm{keV}$ energy range. The similar variation in flux in the $100-300 \mathrm{keV}$ energy range is observed from $\sim 17: 20: 30 \mathrm{UT}$, with a time lag of about $10 \mathrm{~s}$. The electron flux shows a large spatial (or temporal) variation; for example, the flux in the $30-100 \mathrm{keV}$ energy range varies by two orders of magnitude per $0.2^{\circ}$ magnetic latitude (per $4 \mathrm{~s}$ ) from 17:20:16 to 17:20:20 UT.

Figure 3 shows the electron energy spectra derived from the TED and MEPED data at four selected times, as indicated 
28 February, 1998
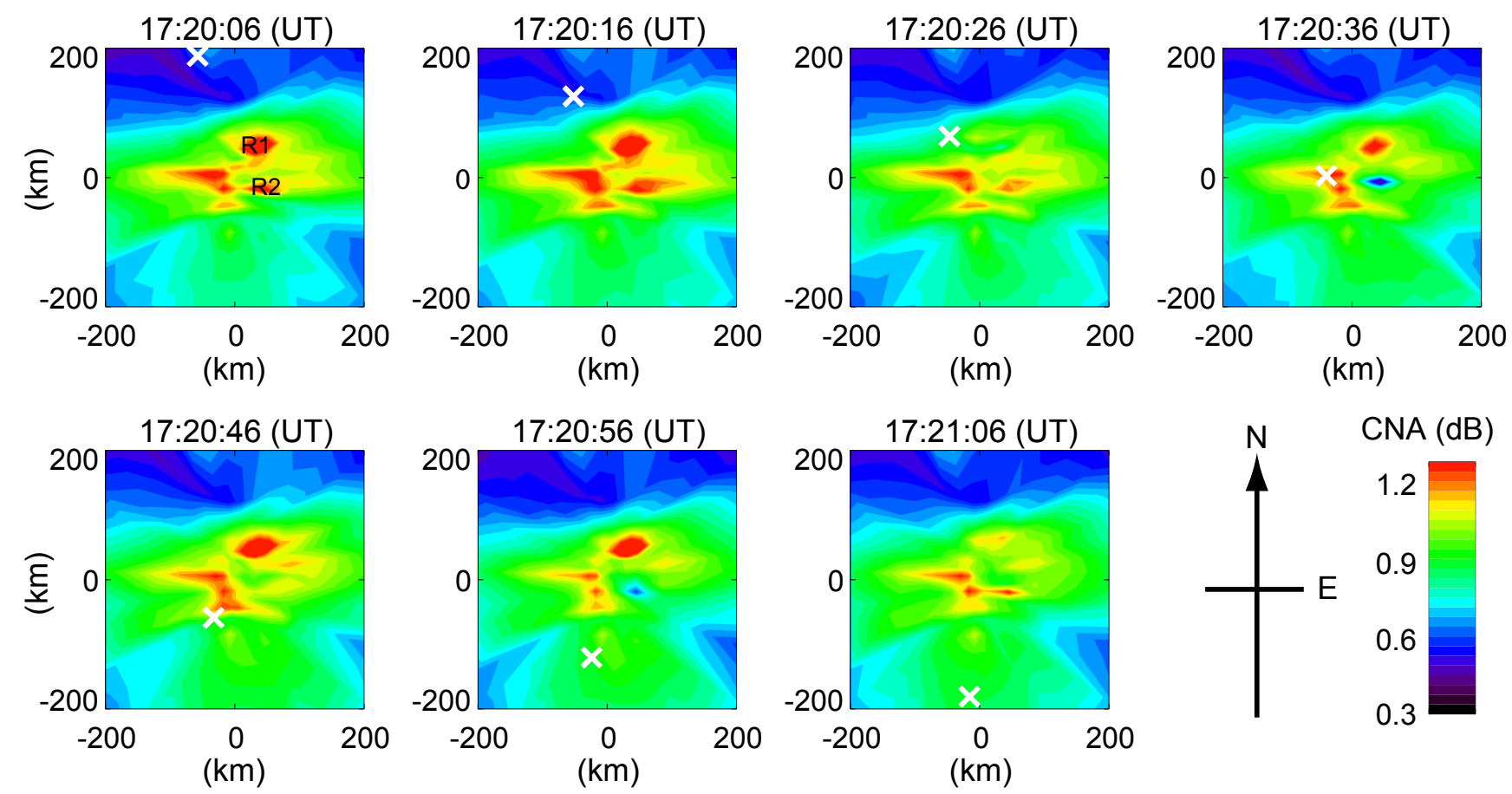

Fig. 1. CNA event observed during 17:20:06-17:21:08 UT on 28 February 1998. The magnetic north and east correspond to the top and right, respectively, as one sees the ionosphere from above. The white cross shows the footprint of NOAA 12 at $90 \mathrm{~km}$ and the scintillations labeled by the letters, $\mathrm{R}_{1}$ and $\mathrm{R}_{2}$, are radio stars, Cassiopeia- $\mathrm{A}$ and Cygnus- $\mathrm{A}$, respectively.

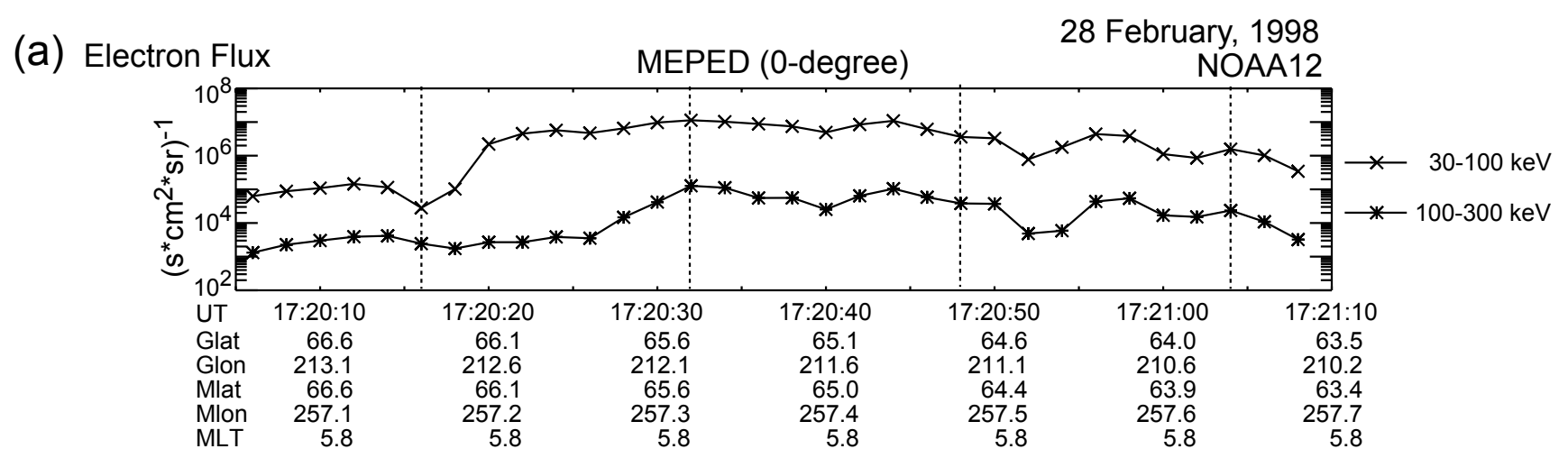

(b) CNA

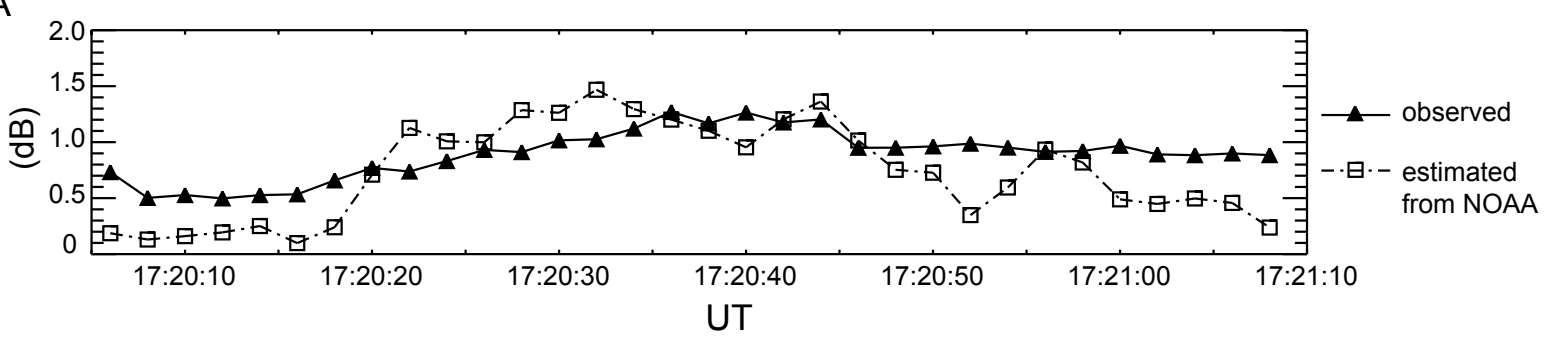

Fig. 2. (a) Electron flux measured at $0^{\circ}$ zenith angle by the MEPED on board NOAA 12 for the event shown in Fig. 1. (b) CNA observed with the imaging riometer on the beam closest to the satellite footprint at $90 \mathrm{~km}$ (solid triangles with the solid line) and the CNA estimated from the precipitating electron flux measured by the TED and MEPED (open squares with the dashed-and-dotted line). 
Table 1. The events selected to evaluate the correlation coefficient between observed and estimated CNA. We selected the eight events for which both the observed and estimated CNA had a range of variation larger than $0.4 \mathrm{~dB}$. The $\mathrm{N}$ indicates the number of data points of each event. The $r_{0}\left(r_{90}\right)$ shows the correlation coefficient between observed and estimated CNA from the precipitating (trapped) electron flux.

\begin{tabular}{cccccccccc}
\hline No. & Date & UT & $\begin{array}{c}\text { MLT } \\
\text { (hour) }\end{array}$ & $\begin{array}{c}K_{p} \\
\text { (nin, Max values } \\
\text { ofserved CNA } \\
(\mathrm{dB})\end{array}$ & $\begin{array}{c}\text { Min, Max values } \\
\text { of estimated CNA } \\
(\mathrm{dB})\end{array}$ & N & $r_{0}$ & $\left(r_{90}\right)$ \\
\hline 1 & 6 October 1995 & $18: 18: 43-18: 19: 45$ & 7.1 & $3-$ & $1.06,1.47$ & $0.35,1.03$ & 26 & -0.12 & $(-0.58)$ \\
2 & 20 March 1996 & $18: 03: 24-18: 04: 26$ & 6.7 & 5 & $0.41,0.92$ & $0.07,1.00$ & 25 & -0.74 & $(0.43)$ \\
3 & 25 March 1996 & $17: 54: 26-17: 55: 28$ & 6.7 & 4 & $0.70,1.29$ & $0.10,1.53$ & 22 & 0.20 & $(0.72)$ \\
4 & 9 February 1997 & $17: 42: 38-17: 43: 40$ & 6.2 & 4 & $0.69,1.25$ & $0.29,1.48$ & 28 & 0.42 & $(0.80)$ \\
5 & 21 September 1997 & $17: 34: 30-17: 35: 32$ & 6.4 & $2+$ & $0.69,1.46$ & $0.08,1.44$ & 25 & 0.81 & $(0.69)$ \\
6 & 19 February 1998 & $17: 17: 56-17: 19: 00$ & 5.8 & $1+$ & $0.21,0.67$ & $0.02,0.89$ & 29 & 0.70 & $(0.70)$ \\
7 & 28 February 1998 & $17: 20: 06-17: 21: 08$ & 5.9 & $3+$ & $0.50,1.27$ & $0.10,1.47$ & 32 & 0.74 & $(0.91)$ \\
8 & 10 April 1998 & $17: 18: 24-17: 19: 26$ & 6.0 & 4 & $1.63,3.15$ & $0.49,2.71$ & 26 & 0.27 & $(0.75)$ \\
\hline
\end{tabular}

by the vertical dotted lines in Fig. 2a. The thick horizontal lines show the measurements used to estimate the energy spectra and the dotted lines show the double Maxwellian fit to the measurements. The thin horizontal lines are derived from additional measurements from four energy channels of the TED, which were not used for the energy spectra estimation, because they were sampled every $8 \mathrm{~s}$. This figure shows that the electron flux can be roughly fitted with a double Maxwellian distribution. The energy spectrum is relatively harder in the enhanced CNA region at 17:20:32 UT than at the other times.

The CNA estimated from the precipitating electron flux is plotted by the open squares in Fig. 2b. The estimated CNA shows a similar trend to the observed CNA, that is, an enhancement during 17:20:20-17:20:44 UT and a depression during 17:20:06-17:20:16 UT and 17:20:48-17:21:08 UT. However, the estimated CNA varies by a larger amount than the observed CNA. The magnitudes of the observed and estimated CNA are comparable around the maximum during 17:20:36-17:20:44 UT, while the estimated CNA is smaller than the observed one by a factor of 2 to 5 during 17:20:0617:20:18 UT and 17:21:00-17:21:08 UT. The correlation coefficient between the observed and estimated CNA is 0.74 .

The above data analysis was made for all the selected 19 events. The correlation coefficient between the observed and estimated CNA must be evaluated carefully, because it is often unreliable when the CNAs do not have a significantly large range of variation. Therefore, we focused on the eight events for which both the observed and estimated CNA had a range of variation larger than $0.4 \mathrm{~dB}$. These events are listed in Table 1. The correlation coefficient $\left(r_{0}\right)$ was greater than 0.7 for three of the eight events (No. 5, 6, 7), while it was less than 0.5 for the rest of the events. For all these events, the minimum estimated CNA is much smaller than the minimum observed CNA, which corresponds to the underestimate shown in Fig. 2b. On the other hand, the maximum observed and estimated values are comparable.

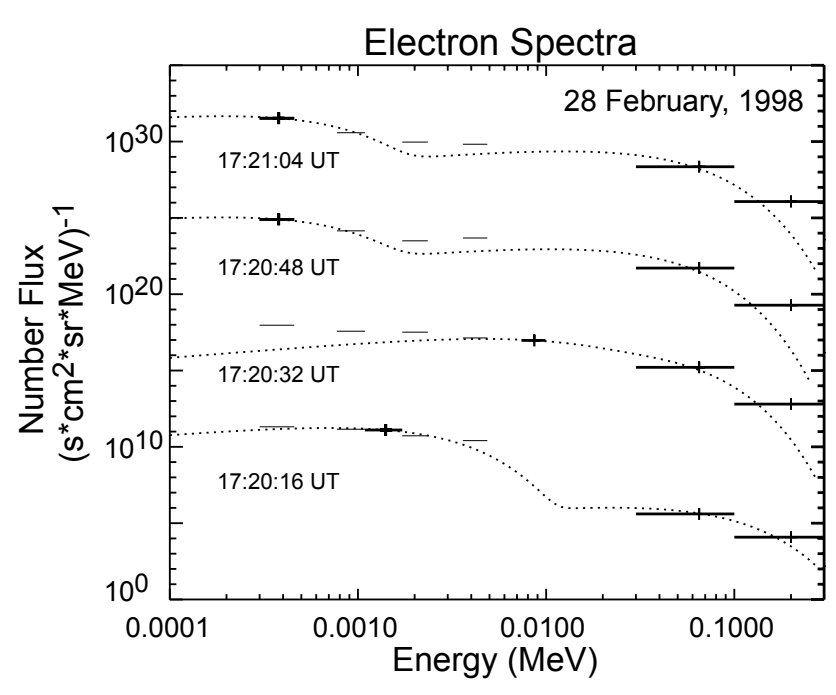

Fig. 3. Energy spectra of precipitating electrons measured by NOAA 12 at four selected times, indicated by the vertical dotted lines in Fig. 2a. The thick horizontal lines show the electron flux used to estimate the energy spectrum and the dotted curves have a double Maxwellian fit to the measurements. The thin horizontal lines are measurements from four energy channels of the TED, which were sampled every $8 \mathrm{~s}$ and were not used to estimate the spectra. The spectra are displaced from one another by 7 orders of magnitude.

It is important to note that the correlation concerns the spatial structures of observed and estimated CNA, if the temporal variation of CNA is small during the passage of the satellite. For example, the CNA event shown in Fig. 1 appears to be stable during the passage of NOAA 12, so Fig. 2 represents the spatial structures of $\mathrm{CNA}$ and the precipitating electron flux rather than the temporal variations. Such correlation analysis of the spatial structures of CNA and the electron flux is a new approach that has never been done before. 


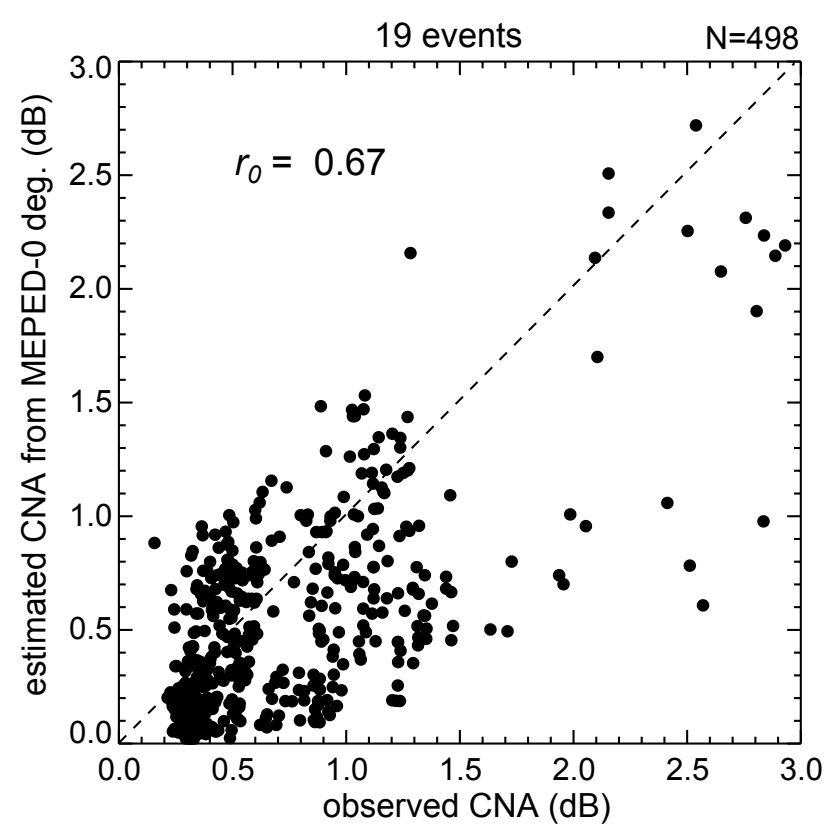

Fig. 4. Scatter plot of CNA estimated from precipitating flux measured by NOAA 12 versus CNA observed with imaging riometer for all 19 events. Each dot corresponds to the CNA sampled every $2 \mathrm{~s}$ for each event. The dashed line shows the ideal regression line, on which the estimated CNA equals the observed one.

To investigate the relationship between the observed and estimated CNA statistically, a scatter plot for all the selected 19 events is presented in Fig. 4. Each dot corresponds to the estimated CNA plotted against the observed CNA that was sampled every $2 \mathrm{~s}$ for each event. The dashed line shows an ideal regression line, on which the estimated CNA equals the observed one. This figure shows a positive correlation between the observed and estimated CNA $\left(r_{0}=0.67\right)$. The estimated CNA has a tendency to be smaller than the observed CNA. The percentage of underestimated events (the dots below the dashed line) is $72 \%$ of the total.

\section{Discussion}

As for the cause of the underestimated CNA, we first examine the anisotropic pitch angle distribution of the electron flux. Figure 5a illustrates the trapped electron flux measured by the MEPED- $90^{\circ}$ for the same event as described in Sect. 4. The format of this figure is same as Fig. 2. The precipitating electron flux from the MEPED- $0^{\circ}$ shown in Fig. $2 \mathrm{a}$ is also plotted using dashed (30-100 keV) and dotted (100$300 \mathrm{keV}$ ) lines. Compared with the precipitating electron flux, the trapped electron flux shows a smoother trend and greater values over the entire interval. Focusing on the 30$100 \mathrm{keV}$ energy range, which is thought to be closely related to the generation of CNA, an anisotropic pitch angle distribution is evident during 17:20:06-17:20:18 UT and 17:20:5217:21:08 UT. The two directional fluxes in the $30-100 \mathrm{keV}$ energy range differ by 1.4 orders of magnitude at maximum at 17:20:16 UT. It is important that the anisotropic distribution corresponds to the period of the underestimate of CNA in Fig. 2b. On the other hand, a nearly isotropic pitch angle distribution was observed during 17:20:20-17:20:48 UT. The anisotropic pitch angle distribution in this figure is consistent with the previous studies (Parthasarathy et al., 1966; Fritz, 1970; Rossberg, 1978; Collis et al., 1983, 1984). Parthasarathy et al. (1966) have shown, using measurements from a low-altitude satellite, that the trapped electron flux with energy $>40 \mathrm{keV}$ observed in the auroral region is typically greater by one to two orders of magnitude than the precipitating electron flux.

To evaluate the influence of the anisotropic pitch angle distribution on CNA quantitatively, we estimated CNA using the trapped electron flux from the MEPED-90 ${ }^{\circ}$. We assumed again an isotropic pitch angle distribution for a significant case. The flux data from the TED- $0^{\circ}$ was used to estimate the low-energy component of energy spectra, because there was no TED detector for the trapped electrons and the low-energy component of the spectra did not have a great influence on the estimated CNA. Figure 5b shows the CNA estimated from the trapped electron flux, together with the observed CNA. The underestimate of CNA in Fig. 2b (in the 17:20:06-17:20:18 UT and 17:20:52-17:21:08 UT intervals) approached the measurements in this figure. In contrast to the precipitating electron flux, the estimated CNA from the trapped electron flux tends to be greater than the observed CNA. The correlation coefficient between the observed and estimated CNA is 0.91, which is higher than the case of the precipitating flux.

The correlation coefficient between the observed and estimated CNA from the trapped electron flux is also shown in Table 1 by $r_{90}$. The correlation coefficient became greater than 0.7 for three of the five low-correlation events described in Sect. 4 (No. 3, 4, 8).

Figure 6 shows a scatter plot of the observed versus estimated CNA from the trapped electron flux for all 19 events. In this figure, a remarkably high correlation can be seen between the observed and estimated CNA $\left(r_{90}=0.87\right)$, which is much higher than the case of the precipitating flux (Fig. 4). Eighty-seven percent of the total events are overestimated, in contrast to Fig. 4.

The underestimate (overestimate) of CNA derived from the precipitating (trapped) electron flux can be interpreted in terms of the anisotropic pitch angle distribution. From an inspection of the MEPED- $90^{\circ}$ flux data, it is likely that the magnetic field line at a $90-\mathrm{km}$ altitude over PFRR $\left(\sim 65^{\circ}\right.$ invariant latitude) was located inside the trapping region of the electrons with energy $>30 \mathrm{keV}$ for all the selected events. This idea is consistent with the previous reports that the high-latitude boundary of the trapped electrons with energy $>30 \mathrm{keV}$ is situated at higher than $70^{\circ}$ invariant latitude in the dawn sector (Fritz, 1970; Rossberg, 1978). Therefore, the pitch angle distribution is expected to be like the loss cone distribution for the anisotropic cases. Since the flux in the loss cone distribution is nearly minimal at $\alpha=0^{\circ}$ (nearly maximal at $\alpha=90^{\circ}$ ), the CNA estimated from the precipitating 


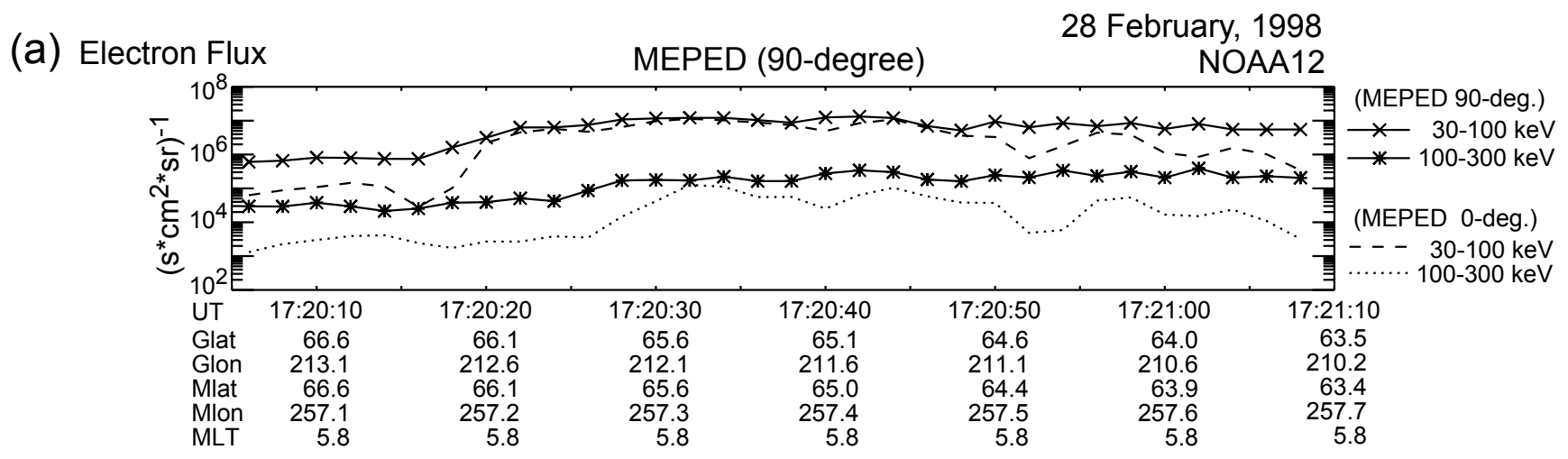

(b) CNA

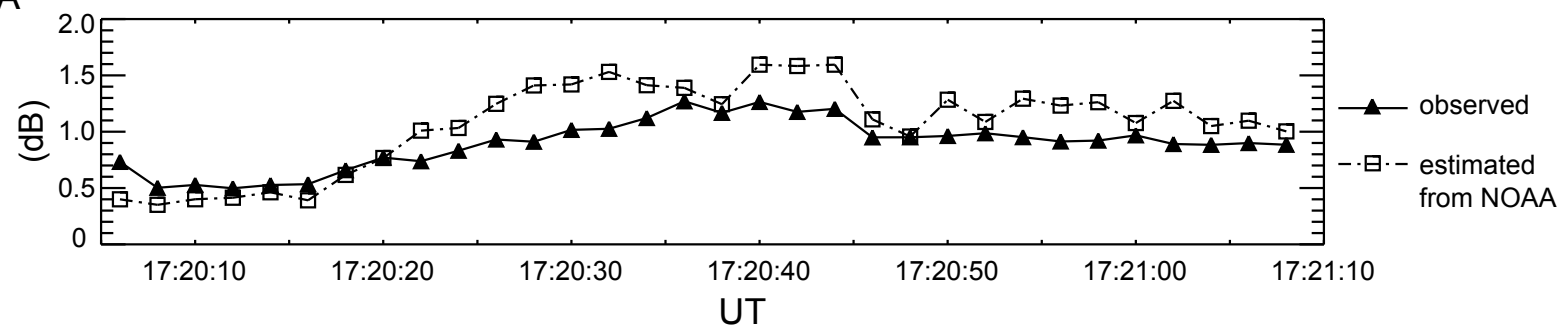

Fig. 5. (a) Trapped electron flux measured by the MEPED- $90^{\circ}$ for the same event as shown in Fig. 2. The dashed (30-100 keV) and dotted $\left(100-300 \mathrm{keV}\right.$ ) lines are the precipitating electron fluxes measured by the MEPED- $0^{\circ}$. (b) The CNA observed with the imaging riometer (solid triangles with the solid line) and CNA estimated using the trapped electron flux measured by the MEPED-90 (open squares with the dashed-and-dotted line). The pitch angle distribution is assumed to be isotropic for the calculation.

(trapped) flux under the assumption of the isotropic distribution is nearly the minimum (maximum) estimate. The CNA expected from the actual pitch angle distribution should be between the two estimates, which is consistent with our results.

The high correlation between the observed and the estimated CNA from the trapped flux $\left(\alpha=65-95^{\circ}\right)$ can also be explained by an anisotropic pitch angle distribution similar to the loss cone distribution. It is believed that the auroral electron precipitation in the morning sector is primarily caused by the pitch angle diffusion of the trapped electrons injected in the substorm, mainly due to the wave-particle interaction (see a review of Davidson, 1990). If the electron flux has a loss cone distribution, electrons which are pitch-angle diffused into the loss cone and the resultant CNA will be correlated with the trapped electron population. It may be possible that the observed CNA is better correlated with the trapped flux $\left(\alpha=65-95^{\circ}\right)$ rather than the small pitch angle flux $(\alpha=0$ $25^{\circ}$ ), because the loss cone angle is approximately $59^{\circ}$ at an 815-km altitude over PFRR and is considerably larger than the aperture of the MEPED- $0^{\circ}$. In such a situation, the large pitch angle flux within the loss cone $\left(\alpha=25-59^{\circ}\right)$ may contribute to the generation of CNA relatively more than the small pitch angle flux $\left(\alpha=0-25^{\circ}\right)$.

A few authors have reported CNA events related to increased electron density in the ionospheric F region (Rosenberg et al., 1993; Wang et al., 1994). With radar observations, they showed that the CNA events are associated with electron density patches in the F region, which form in the dayside

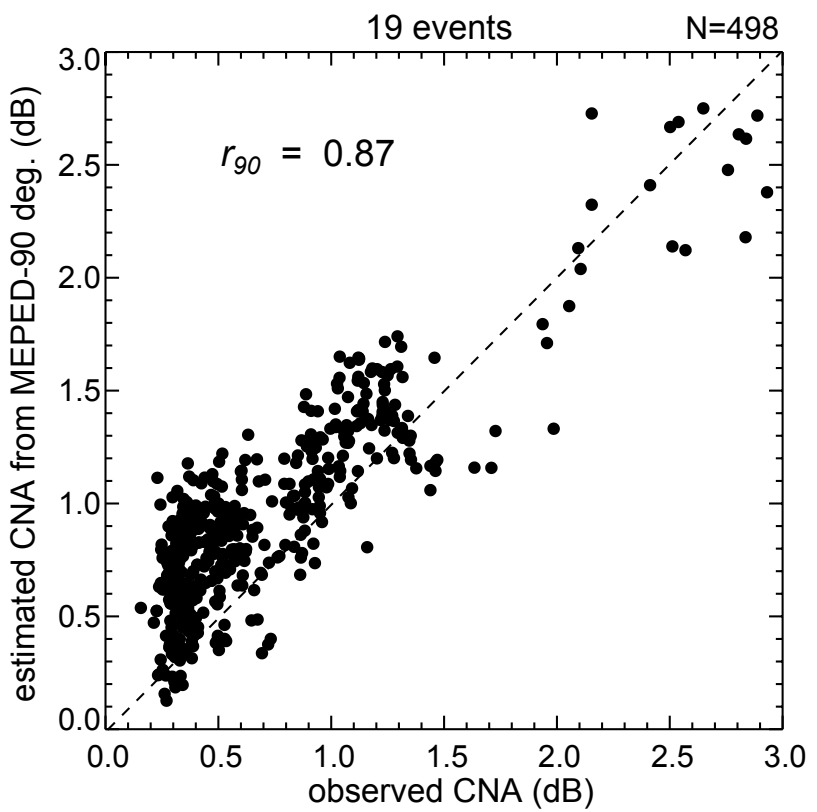

Fig. 6. Scatter plot of observed CNA versus CNA estimated using the trapped electron flux from the MEPED- $90^{\circ}$ for all the selected events. The format of this figure is the same as Fig. 4.

cusp region and drift into the polar cap. Regarding our results, the larger CNA than estimated from the precipitating electron flux (i.e. underestimate of CNA) may be explained by the overlap of the F-region CNA with the D- and E-region 
CNA due to the precipitation. However, the CNA due to the F-region density patches are usually very small and at most $0.3 \mathrm{~dB}$. In addition, the electron density patches are usually observed at higher magnetic latitudes than PFRR and very few occur near the magnetic dawn (Rodger and Graham, 1996). Therefore, the effect of the F-region CNA will be negligible.

We discuss here the uncertainty of CNA by the assumed effective recombination coefficient $\left(\alpha_{e f f}(h)\right)$. We fixed the $\alpha_{e f f}(h)$ profile in estimating CNA, in order to concentrate on the relationship between CNA and the electron flux. However, a significant variation of $\alpha_{e f f}(h)$ is theoretically predicted in the $\mathrm{D}$ region due to the factors such as the season, local time, ionization rate, etc. (e.g. Penman et al., 1979). Collis et al. (1984) examined the effect of $\alpha_{e f f}(h)$ on CNA by using two limits of the $\alpha_{e f f}(h)$ profile, that is, the "upper limiting profile (ULP)" of Penman et al. (1979) and the geometric mean (GMEAN) of their "upper" and "lower" limiting profiles. They found that CNA calculated with the ULP (GMEAN) profile are underestimated (overestimated) by a factor of about two with respect to the observed values. We also estimated the uncertainty of CNA due to $\alpha_{e f f}(h)$ in the same way of Collis et al. (1984). The CNA calculated with the ULP (GMEAN) profile were less (greater) than estimated with the $\alpha_{e f f}(h)$ profile from Hargreaves and Devlin (1990) by a factor of 1.7 (1.3), on average. Therefore, the scatter plots of the observed versus estimated CNA in Figs. 4 and 6 have the possibility to be distributed both above and below the current plots in this range.

Finally, we examine the effect of oblique beams on the observed CNA. The observed CNA has been corrected by simply applying a factor, $\cos (c \chi)$, where $\chi$ is the zenith angle of the beam center and $c$ is the constant determined from measurements of the polar cap absorption (PCA) event. The CNA measured on the oblique beam varies nonlinearly with respect to the true zenithal absorption, due to the effect of sidelobes, as well as the shape of the main beam (Hargreaves and Detrick, 2002). We have examined the dependence of the observed to estimated CNA ratio on the zenith angle of the beam center (not shown here). The results showed that the ratio has little dependence on the zenith angle, therefore, the effect of the oblique beams on the CNA does not cause the underestimate.

\section{Summary and conclusions}

In this paper, we compared CNA with the precipitating electron flux for 19 events observed in the morning sector, by using the high-resolution data obtained during conjugate observations with the imaging riometer at PFRR, Alaska, and the low-altitude satellite, NOAA 12 . We traced exactly the magnetic field line from NOAA 12 to the footprint at $90 \mathrm{~km}$ and selected CNA data measured on the beam closest to the footprint. According to a theoretical model assuming an isotropic pitch angle distribution, the CNA was estimated from the precipitating electron flux measured by NOAA 12 and was com- pared with the CNA observed by the imaging riometer. The high-resolution data enabled us to examine the correlation between the observed and estimated CNA for the individual events corresponding to the individual passages of the satellite.

We focused on the eight events for which both the observed and estimated CNA had a range of variation larger than $0.4 \mathrm{~dB}$ and found that three events showed high correlation between observed and estimated CNA $(r>0.7)$ and five showed low correlation $(r<0.5)$. The estimated CNA tended to be smaller than the observed CNA ( $72 \%$ of all data for 19 events) and the underestimate of CNA appeared to be the main cause of the low-correlation events. The influence of the anisotropic pitch angle distribution on CNA was examined using the trapped electron flux measured by the MEPED-90. The resulting CNA, assuming an isotropic pitch angle distribution, was highly correlated with the observed CNA and tended to be overestimated $(87 \%$ of all data). The underestimate (overestimate) of CNA derived from the precipitating (trapped) electron flux can be interpreted in terms of an anisotropic pitch angle distribution similar to a loss cone distribution.

Our analysis reduced the ambiguity in the relationship between CNA and the electron flux, caused by the discrepancy in the locations of the absorption and the satellite footprint. Our results indicate that the pitch angle distribution and loss cone angle of the electrons are important factors to correlate CNA with precipitating electrons. CNA observed with a riometer may be quantitatively explained with a model based on energetic electron precipitation from the magnetosphere, provided that the pitch angle distribution and loss cone angle of the electrons are taken into account.

Acknowledgements. This study has been supported in part by the U.S.-Japan international research project to observe the middle atmosphere, NICT, Japan. The authors wish to thank the technical staff of the Geophysical Institute of University of Alaska Fairbanks (GI/UAF) for assisting in operation of the imaging riometer at Poker Flat. The $A E$ and $K_{P}$ indices were provided by the WDC-C2 for Geomagnetism at Kyoto University, Japan.

Topical Editor U.-P. Hoppe thanks M. Friedrich, P. Stocker and another referee for their help in evaluating this paper.

\section{References}

Codrescu, M. V., Fuller-Rowell, T. J., Roble, R. G., and Evans, D. S.: Medium energy particle precipitation influences on the mesosphere and lower thermosphere, J. Geophys. Res., 102, 1997719987, 1997.

Collis, P. N., Hargreaves, J. K., and Korth, A.: Loss cone fluxes and pitch angle diffusion at the equatorial plane during auroral radio absorption events, J. Atmos. Terr. Phys., 45, 231-243, 1983.

Collis, P. N., Hargreaves, J. K., and Korth, A.: Auroral radio absorption as an indicator of magnetospheric electrons and of conditions in the disturbed auroral D-region, J. Atmos. Terr. Phys., 46, 21-38, 1984.

Collis, P. N. and Korth, A.: GEOS-2 observations of energetic electrons in the morning sector during auroral radio absorption events, J. Atmos. Terr. Phys., 47, 327-339, 1985. 
Davidson, G.: Pitch-angle diffusion and the origin of temporal and spatial structures in morningside aurorae, Space Sci. Rev., 53, 45-82, 1990.

Fritz, T. A.: Study of the high-latitude, outer-zone boundary region for $\geq 40 \mathrm{kev}$ electrons with satellite Injun 3, J. Geophys. Res., 75, 5387-5400, 1970.

Garrett, H. B., Schwank, D. C., and Deforest, S. E.: A statistical analysis of the low-energy geosynchronous plasma environment - I. electrons, Planet. Space Sci., 29, 1021-1044, 1981.

Hargreaves, J. K. and Sharp, R. D.: Electron precipitation and ionospheric radio absorption in the auroral zones, Planet. Space Sci., 13, 1171-1183, 1965.

Hargreaves, J. K. and Devlin, T.: Morning sector electron precipitation events observed by incoherent scatter radar, J. Atmos. Terr. Phys., 52, 193-203, 1990.

Hargreaves, J. K. and Detrick, J. K.: Application of polar cap absorption events to the calibration of riometer systems, Radio Science, 37, 1035-1045, 2002.

Hartz, T. R. and Brice, N. M.: The general pattern of auroral particle precipitation, Planet. Space Sci., 15, 301-329, 1967.

Jackman, C. H., Frederick, J. E., and Stolarski, R. S.: Production of odd nitrogen in the stratosphere and mesosphere: an intercomparison of source strengths, J. Geophys. Res., 85, 7495-7505, 1980 .

Jelly, D. and Brice, N.: Changes in Van Allen radiation associated with polar substorms, J. Geophys. Res., 72, 5919-5931, 1967.

Maehlum, B. and O'Brien, B. J.: Study of energetic electrons and their relationship to auroral absorption of radio waves, J. Geophys. Res., 68, 997-1010, 1963.

McDiarmid, I. B., Burrows, J. R., and Budzinski, E. E.: Average characteristics of magnetospheric electrons $(150 \mathrm{eV}$ to $200 \mathrm{keV})$ at $1400 \mathrm{~km}$, J. Geophys. Res., 80, 73-79, 1975.
Murayama, Y., Mori, H., Kainuma, S., Ishii, M., Nishimuta, I., Igarashi, K., Yamagishi, H., and Nishino, M.: Development of a high-resolution imaging riometer for the middle and upper atmosphere observation program at Poker Flat, Alaska, J. Atmos. Terr. Phys., 59, 925-937, 1997.

Parthasarathy, R., Berkey, F. T., and Venkatesan, D.: Auroral zone electron flux and its relation to broadbeam radiowave absorption, Planet. Space Sci., 14, 65-83, 1966.

Penman, J. M., Hargreaves, J. K., and McIlwain, C. E.: The relation between 10 to $80 \mathrm{keV}$ electron precipitation observed at geosynchronous orbit and auroral radio absorption observed with riometers, Planet. Space Sci., 27, 445-451, 1979.

Raben, V. J., Evans, D. S., Sauer, H. H., Sahm, S R., and Huynh, M.: TIROS-NOAA satellite space environment monitor data archive documentation: 1995 update, NOAA Tech. Memo. ERL SEL-86, 1995.

Rodger, A. S. and Graham, A. C.: Diurnal and seasonal occurrence of polar patches, Ann. Geophys., 14, 533-537, 1996,

SRef-ID: 1432-0576/ag/1996-14-533.

Rosenberg, T. J., Wang, Z., Rodger, A. S., Dudeney, J. R., and Baker, K. B.: Imaging riometer and HF radar measurements of drifting $\mathrm{F}$ region electron density structures in the polar cap, J. Geophys. Res., 98, 7757-7764, 1993.

Rossberg, L.: Undisturbed trapping boundary for energetic electrons at low altitudes, J. Geophys. Res., 83, 4307-4317, 1978.

Semeter, J., Lummerzheim, D., and Haerendel, G..: Simultaneous multispectral imaging of the discrete aurora, J. Atmos. Terr. Phys., 63, 1981-1992, 2001.

Wang, Z., Rosenberg, T. J., Stauning, P., Basu, S., and Crowley, G..: Calculations of riometer absorption associated with $\mathrm{F}$ region plasma structures based on Sondre Stromfjord incoherent scatter radar observations, Radio Science, 29, 209-215, 1994. 